\title{
Association between the expression of secreted phosphoprotein - related genes and prognosis of human cancer
}

\author{
Yaqin Tu', Cai Chen ${ }^{2}$ and Guorun Fan ${ }^{1 *}$ (D)
}

\begin{abstract}
Background: While many studies have assessed the predictive value of secreted phosphoprotein (SPP) genes in cancer, the findings have been inconsistent. To resolve these inconsistencies, we systematically analyzed the available data to determine whether SPP1 and SPP2 are prognostic markers in the context of human cancer.

Methods: The expression of SPP1 and SPP2 was assessed by Oncomine analysis. The PrognoScan database was used to assess the prognostic value of SPP1 and SPP2, with cBioPortal used to assess copy number variations. The STRING database was used to generate a Protein - Protein Interaction (PPI) network for SPP genes.

Results: SPP1 was more likely to be over-expressed in breast, bladder, colorectal, head, neck, liver, lung, and esophageal cancers. SPP2 was expressed at lower levels in colorectal cancer, leukemia, liver cancer and pancreatic cancer. In addition, SPP1 and SPP2 mutations mainly occurred in cutaneous melanoma and endometrial cancer.

Conclusions: Our results suggest that SPP1 and SPP2 may be effective therapeutic or diagnostic targets in certain cancers. Further research is required to confirm these results and verify the value of SPP1 and SPP2 as clinical markers of cancer prognosis.
\end{abstract}

Keywords: Secreted phosphoprotein 1, Secreted phosphoprotein 2, Expression, Prognosis, Human cancer

\section{Background}

Cancer is one of the most serious diseases threatening human health and has become a major public health problem [1]. Cancers are heterogeneous in nature; each type of cancer is associated with many unique epigenetic and genetic variations [2]. Studies exploring the processes of tumor development and those that investigate specific cancer expression profiles offer invaluable insight into both the molecular underpinnings of the disease and the potential diagnostic and therapeutic targets for use in patients [3]. Irreparable structural mutations in cells are the main cause of human cancer; these alter the DNA copy number and function of a gene at a very specific genomic location. Identifying copy number alterations is a useful approach for linking copy number alterations (CNAs) with the disease phenotype. Thus, the current

\footnotetext{
*Correspondence: guorun2006@126.com

'Department of Otorhinolaryngology, Union Hospital, Tongji Medical College, Huazhong University of Science and Technology, Wuhan 430022, China Full list of author information is available at the end of the article
}

study offers cell-level insight into the genetic and epigenetic changes influencing the altered biochemical processes observed in tumor cells.

Secreted phosphoprotein 1 (SPP1), also named Osteopontin (OPN), is an integrin-binding protein that is secreted from various types of cells, including macrophages, endothelial cells, and osteoclasts. In humans, SPP1 is composed of 6 introns and 7 exons, and is encoded on chromosome 4 (4q13) [4]. SPP1 is involved in multiple physiological and pathological processes. Recent studies have reported that SPP1 is significantly associated with cell growth, adherence and invasion in tumourigenesis and metastasis, and is over-expressed in lung [5], colon [6], breast [7], and prostate cancers [8]. The expression level of SPP1 correlates with tumor stage and aggressiveness, suggesting that OPN may be a diagnostic and prognostic biomarker for several cancers. On the other hand, secreted phosphoprotein 2 (SPP2) is a bone matrix protein that can bind to and inhibit the bone morphogenetic proteins (BMPs) inducing bone 
formation. The SPP2 gene spans approximately $27 \mathrm{~kb}$ at chromosome 2 (2q37.1) and encodes secreted phosphoprotein $24 \mathrm{kD}$ [9]. Cancers are often associated with misregulation of the BMP signaling pathway. Previous studies have shown that SPP2 inhibits the growth of tumor cells in prostate cancer [10], pancreatic cancer [9] and hepatocellular carcinoma [11] and attenuates the growth-enhancing effects of BMP2. Thus, we hypothesize that SPP plays an oncogenic or antioncogenic function in different cancers. To explore the character of SPP members in cancers, oncomine platform assesses the gene expression of cancer by 86 , 733 microarray experiments. Furthermore, the survival of cancer patients was analyzed by PrognoScan database. The co-expression data revealed the biological function and provided insight into the potential underlying mechanism. The gene ontology enrichment by STRING is able to discover the function and regulatory mechanism of genes. Basing on many available database results pertaining to changes in gene expression or copy number, we conducted a deep analysis of alterations in SPP gene expression or copy number in the tumors of cancer patients. The goal of this analysis was to understand how the expression and mutation of these genes are associated with patient outcomes.

\section{Materials and methods Oncomine data analysis}

Data sets available within the Oncomine database (https://www.oncomine.org), which compiles previously published microarray data, were employed in order to assess SPP expression patterns in different types of cancer. For each dataset we assessed comparisons of mRNA expression between tumor and normal tissue based on the following thresholds: $p$-value $<1 \mathrm{E}-4$, fold change $>2$. We only identified the top $10 \%$ of differentially expressed genes, and using the compiled data we generated heat maps of differential SPP gene expression in different cancer types.

\section{Prognoscan database analysis}

Using the PrognoScan database (http://dna00.bio.kyutech. ac.jp/PrognoScan/), we assessed the relationsip between SPP gene expression and survival in different cancer types, using a cox p-value threshold of $<0.05$ [12].

\section{Protein-protein interaction (PPI) network construction}

In order to better understand molecular mechanisms governing carcinogenesis, we employed the STRING database to generate a PPI network for SPP genes. We used a minimum interaction score of at least 0.4 as a cut-off when visualizing this network.

\section{cBioPortal database analysis}

We additionally employed the open-access cBioPortal for Cancer Genomics database, which is available to assist with visualization and interpretation of large cancer genomic data (http://www.cbioportal.org/) [13, 14]. We were thereby able to review records from 215 separate studies covering 31 cancers and over 66,000 total samples. Our main parameters for exploring RNA-seq datasets with this database included SPP gene alterations (amplifications, deletions, or missense mutations) and Copy Number alterations (CNAs).

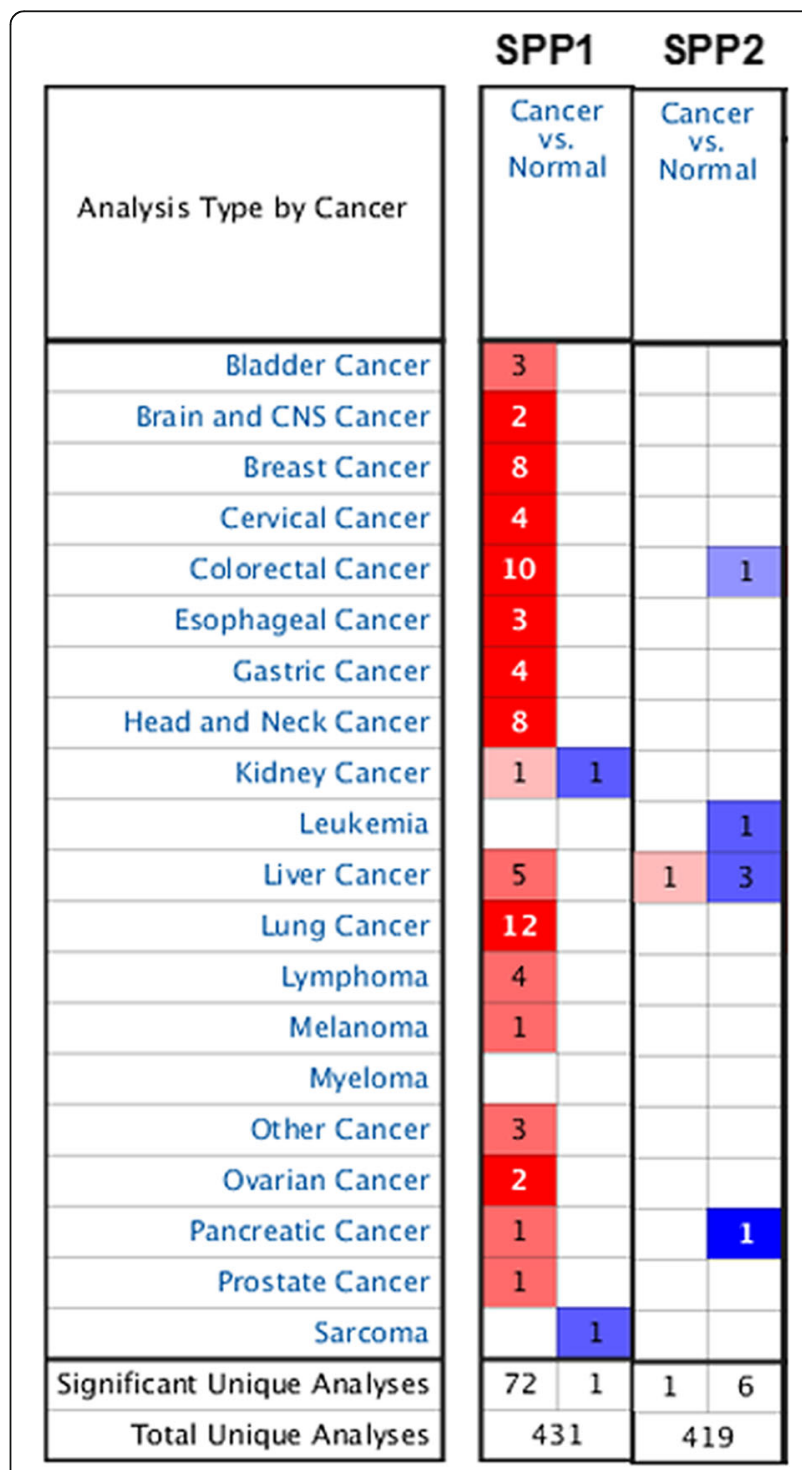

Fig. 1 The transcription levels of SPP1 and SPP2 in different types of cancers, This graphic was generated from Oncomine, indicating the numbers of datasets with statistically significant mRNA over-expression (Red) or down-expression (Blue) of SPP1 and SPP2 in cancer versus normal tissue.The threshold was designed with following parameters: $\mathrm{p}$-value of $1 \mathrm{E}-4$, fold change of 2, and gene ranking of $10 \%$ 


\section{Statistical analysis}

All results are displayed with $p$ values from a log-tank test. Survival curves were generated by the PrognoScan database, using a cox $p$-value threshold of $<0.05$. Statistical significance of the data ( $p$-values) was provided by the program.

\section{Results}

\section{The expression of SPP1 and SPP2 in various cancers}

To assess the importance of SPP in various cancers, SPP1 and SPP2 expression were analyzed in healthy and tumor tissues via the Oncomine database. We found that SPP1 was upregulated in breast, bladder, colorectal, head and neck, liver cancer, lung, and esophageal cancers, whereas decreased in kidney cancer and sarcoma $(p<$ 0.05 , Fig. 1 and Table 1). SPP2 was under-expressed in colorectal cancer, leukemia, liver cancer and pancreatic cancer $(p<0.05$, Fig.1 and Table 2). We additionally utilized Oncomine database to confirm SPP expression in various forms of cancer $(p<0.05$, Fig.2 and Fig.3). We found that in certain cancers SPP1 was over-expressed, while in others it was under-expressed, suggesting that depending on the particular cancer type SPP1 may be playing a pro- or anti-oncogneic function. However, SPP2 is generally lowly expressed in tumors, suggesting that SPP2 may serve as tumor suppressor gene.

\section{SPP expression is associated with survival in various cancers}

Using a Prognostic database, we assessed the predictive link between SPP expression and patient survival in

Table 1 SPP1 expression in cancers

\begin{tabular}{|c|c|c|c|c|c|}
\hline Cancer & Cancer subtype & $P$ value & Fold change & Sample & Reference \\
\hline \multirow[t]{2}{*}{ Lung } & Lung Adenocarcinoma & $6.73 \mathrm{E}-38$ & 20.616 & 107 & $18,297,132$ \\
\hline & Squamous Cell Lung Carcinoma & $5.67 \mathrm{E}-7$ & 60.245 & 203 & $11,707,567$ \\
\hline \multirow[t]{2}{*}{ colon } & Colon Mucinous Adenocarcinoma & 7.35E-11 & 35.194 & 105 & $17,615,082$ \\
\hline & Colon Adenocarcinoma & $1.50 \mathrm{E}-11$ & 13.170 & 105 & $17,615,082$ \\
\hline Cervix & Cervical Squamous Cell Carcinoma & $1.93 \mathrm{E}-15$ & 18.837 & 66 & $18,506,748$ \\
\hline Brain & Glioblastoma & $1.03 \mathrm{E}-6$ & 2.346 & 54 & $16,204,036$ \\
\hline \multirow[t]{3}{*}{ Head-Neck } & Head and Neck Squamous Cell Carcinoma & $1.31 \mathrm{E}-20$ & 43.614 & 54 & $14,729,608$ \\
\hline & Oral Cavity Squamous Cell Carcinoma & $8.04 \mathrm{E}-24$ & 11.215 & 79 & $21,853,135$ \\
\hline & Tongue Squamous Cell Carcinoma & $2.52 \mathrm{E}-9$ & 15.528 & 58 & $19,138,406$ \\
\hline Ovarian & Ovarian Serous Adenocarcinoma & $3.12 \mathrm{E}-5$ & 25.623 & 16 & $14,760,385$ \\
\hline \multirow[t]{2}{*}{ Gastric } & Gastric Cancer & $2.52 \mathrm{E}-10$ & 4.042 & 160 & $20,965,966$ \\
\hline & Gastric Intestinal Type Adenocarcinoma & $2.58 \mathrm{E}-13$ & 15.519 & 96 & $19,081,245$ \\
\hline Esophagus & Esophageal Squamous Cell Carcinoma & $1.99 \mathrm{E}-22$ & 9.154 & 106 & $21,385,931$ \\
\hline \multirow[t]{3}{*}{ Breast } & Invasive Ductal Breast Carcinoma Stroma & 2.87E-5 & 16.337 & 22 & $17,914,389$ \\
\hline & Invasive Breast Carcinoma & 7.36E-6 & 4.068 & 2136 & $22,522,925$ \\
\hline & Tubular Breast Carcinoma & 4.30E-19 & 4.813 & 2136 & $22,522,925$ \\
\hline Bladder & Infiltrating Bladder Urothelial Carcinoma & 1.19E-14 & 6.229 & 157 & $16,432,078$ \\
\hline Liver & Hepatocellular Carcinoma & 1.66E-10 & 7.505 & 115 & $19,098,997$ \\
\hline \multirow[t]{4}{*}{ Lymphoma } & Primary Effusion Lymphoma & $6.97 \mathrm{E}-8$ & 137.979 & 336 & $15,778,709$ \\
\hline & Centroblastic Lymphoma & 2.66E-9 & 12.803 & 336 & $15,778,709$ \\
\hline & Diffuse Large B-Cell Lymphoma & $5.55 E-18$ & 12.096 & 136 & $19,412,164$ \\
\hline & Unspecified Peripheral T-Cell Lymphoma & $1.31 \mathrm{E}-9$ & 8.739 & 60 & $17,304,354$ \\
\hline \multirow[t]{2}{*}{ Prostate } & Prostate Carcinoma & $1.95 \mathrm{E}-5$ & 3.004 & 122 & $22,722,839$ \\
\hline & Prostate Adenocarcinoma & $1.23 \mathrm{E}-4$ & 2.185 & 40 & $12,873,976$ \\
\hline Melanoma & Cutaneous Melanoma & $6.43 \mathrm{E}-8$ & 13.322 & 70 & $18,254,958$ \\
\hline \multirow[t]{2}{*}{ Pancreas } & Pancreatic Ductal Adenocarcinoma & $4.26 \mathrm{E}-11$ & 6.619 & 78 & $16,204,036$ \\
\hline & Pancreatic Intraepithelial Neoplasia & $3.10 \mathrm{E}-5$ & 1.827 & 38 & $16,103,885$ \\
\hline Sarcoma & Myxofibrosarcoma & $1.01 \mathrm{E}-4$ & 6.287 & 40 & $20,601,955$ \\
\hline Kidney & Clear Cell Sarcoma of the Kidney & $3.07 \mathrm{E}-5$ & -3.934 & 35 & $16,299,227$ \\
\hline
\end{tabular}


Table 2 SPP2 expression in cancers

\begin{tabular}{|c|c|c|c|c|c|}
\hline Cancer & Cancer subtype & $P$ value & Fold change & Sample & Reference \\
\hline \multirow[t]{2}{*}{ Breast } & Invasive Lobular Breast Carcinoma & 0.002 & 2.598 & 30 & $17,389,037$ \\
\hline & Mucinous Breast Carcinoma & 0.045 & 6.717 & 593 & TCGA \\
\hline \multirow[t]{2}{*}{ Lung } & Micropapillary Lung Adenocarcinoma & 0.003 & 1.046 & 1537 & TCGA \\
\hline & Lung Adenocarcinoma & $8.83 \mathrm{E}-7$ & 2.341 & 246 & $22,080,568$ \\
\hline Esophagus & Barrett's Esophagus & 0.023 & 1.230 & 52 & $16,449,976$ \\
\hline Prostate & Prostate Carcinoma & $3.36 \mathrm{E}-4$ & 1.137 & 102 & $12,086,878$ \\
\hline Pancreas & Pancreatic Adenocarcinoma & $4.68 \mathrm{E}-5$ & 1.033 & 100 & TCGA \\
\hline \multirow[t]{4}{*}{ Lymphoma } & T-Cell/Histiocyte-Rich Large B-Cell Lymphoma & 0.011 & 1.220 & 67 & $18,794,340$ \\
\hline & Diffuse Large B-Cell Lymphoma & 0.009 & 1.132 & 67 & $18,794,340$ \\
\hline & Burkitt's Lymphoma & 0.031 & 1.195 & 67 & $18,794,340$ \\
\hline & Follicular Lymphoma & 0.035 & 1.167 & 67 & $18,794,340$ \\
\hline \multirow[t]{3}{*}{ Melanoma } & Skin Basal Cell Carcinoma & 0.006 & 2.763 & 87 & $18,442,402$ \\
\hline & Pleural Malignant Mesothelioma & 0.016 & 2.195 & 54 & $15,920,167$ \\
\hline & Skin Squamous Cell Carcinoma & 0.048 & 1.906 & 87 & $18,442,402$ \\
\hline Brain & Primary Glioblastoma & 0.005 & 1.035 & 187 & $18,077,431$ \\
\hline Gastric & Gastric Mixed Adenocarcinoma & 0.008 & 1.070 & 90 & $21,447,720$ \\
\hline Thyroid & Thyroid Gland Oncocytic Follicular Carcinoma & 0.045 & 1.104 & 99 & $16,609,007$ \\
\hline Liver & Hepatocellular Carcinoma & $1.18 \mathrm{E}-42$ & -5.790 & 445 & $21,159,642$ \\
\hline \multirow[t]{2}{*}{ Colon } & Rectal Adenocarcinoma & $3.58 \mathrm{E}-4$ & -1.824 & 237 & TCGA \\
\hline & Colon Adenocarcinoma & $6.61 \mathrm{E}-4$ & -1.658 & 237 & TCGA \\
\hline \multirow[t]{2}{*}{ Ovarian } & Ovarian Mucinous Adenocarcinoma & 0.024 & 1.085 & 103 & $16,452,189$ \\
\hline & Colon Adenocarcinoma & $1.10 \mathrm{E}-5$ & -2.941 & 123 & $17,640,062$ \\
\hline \multirow[t]{2}{*}{ Pancreas } & Pancreatic Carcinoma & 0.003 & -1.411 & 17 & $15,867,264$ \\
\hline & Pancreatic Ductal Adenocarcinoma & $1.88 \mathrm{E}-8$ & -1.368 & 78 & $19,260,470$ \\
\hline
\end{tabular}

various cancer types. Patients who had a high expression of SPP1 showed poor prognosis in melanoma and in blood, brain, breast, colorectal, and lung cancer $(p<$ 0.05, Fig.4, Additional file 1 and Table 3). The overexpression of SPP2 was linked with reduced survival in those with ovarian cancer, whereas it was linked with improved survival in breast and lung cancer patients $(p<0.05$, Fig.5, Additional file 1 and Table 4).

\section{Molecular functional pathways and process of SPP1 and SPP2}

We use the String database to predict the ten proteins that interact with the SPP1 and SPP2, respectively. For SPP1 these included (with the corresponding gene names): Tumor Protein P53 (TP53), Matrix Metallopeptidase 3 (MMP3), Matrix Metallopeptidase 7 (MMP7), CD44, Bone Gamma-Carboxyglutamate Protein (BGLAP), Integrin Subunit Beta 1 (ITGB1), Integrin Subunit Beta 3 (ITGB3), Integrin Subunit Beta 5 (ITGB5), Integrin Subunit Alpha 5 (ITGA5), and Integrin Subunit Alpha V (ITGAV). For SPP2 these included (with the corresponding gene names): Fibrinogen Gamma Chain (FGG), Fibrinogen Alpha Chain
(FGA), Fibrinogen Beta Chain (FGB), Histidine Rich Glycoprotein (HRG), Alpha 2-HS Glycoprotein (AHSG), Plasminogen (PLG), Orosomucoid 1 (ORM1), Orosomucoid 2 (ORM2), Albumin (ALB), and Hepatocyte Growth Factor (HGF). The biological pathways and process were calculated by Funrich software, and PPI network was generated from STRING online tool (Fig.6).

\section{Mutations and copy number alterations of SPP genes in different cancers}

We analyzed SPP1 gene mutations and copy number alterations by via assessing 198 studies using the cBioportal tool. We observed a clear amplification pattern in prostate cancer, whereas mutations in SPP1 primarily occurred in cutaneous melanoma and endometrial cancer. The ratio of alteration ranged from 1.03 to $9.23 \%$ (Fig.7a). For SPP2 gene, an amplification pattern of interest was also observed in prostate cancer. Also, SPP2 mutation was most predominant in cutaneous melanoma and endometrial cancer. The frequency of alteration ranged from 1.06 to $10.77 \%$ (Fig.7b). 

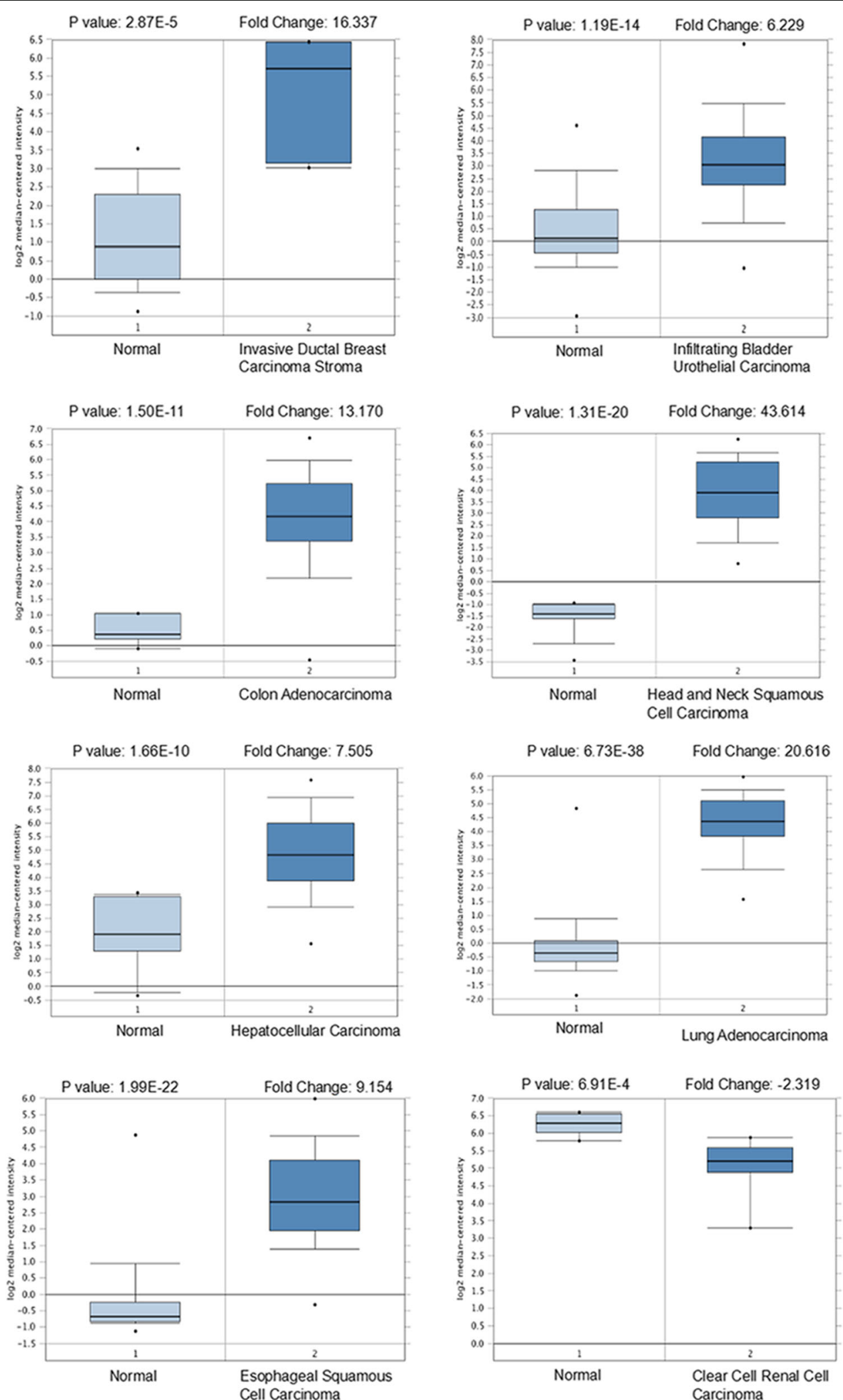

Fig. 2 The expression level of SPP1 in different cancer types (Oncomine database), The box plot comparing specific SPP1 expression in normal (left plot) and cancer tissue (right plot) was derived from Oncomine database. The fold change of SPP1 in various types of cancers was identified from our analyses in Table 1 

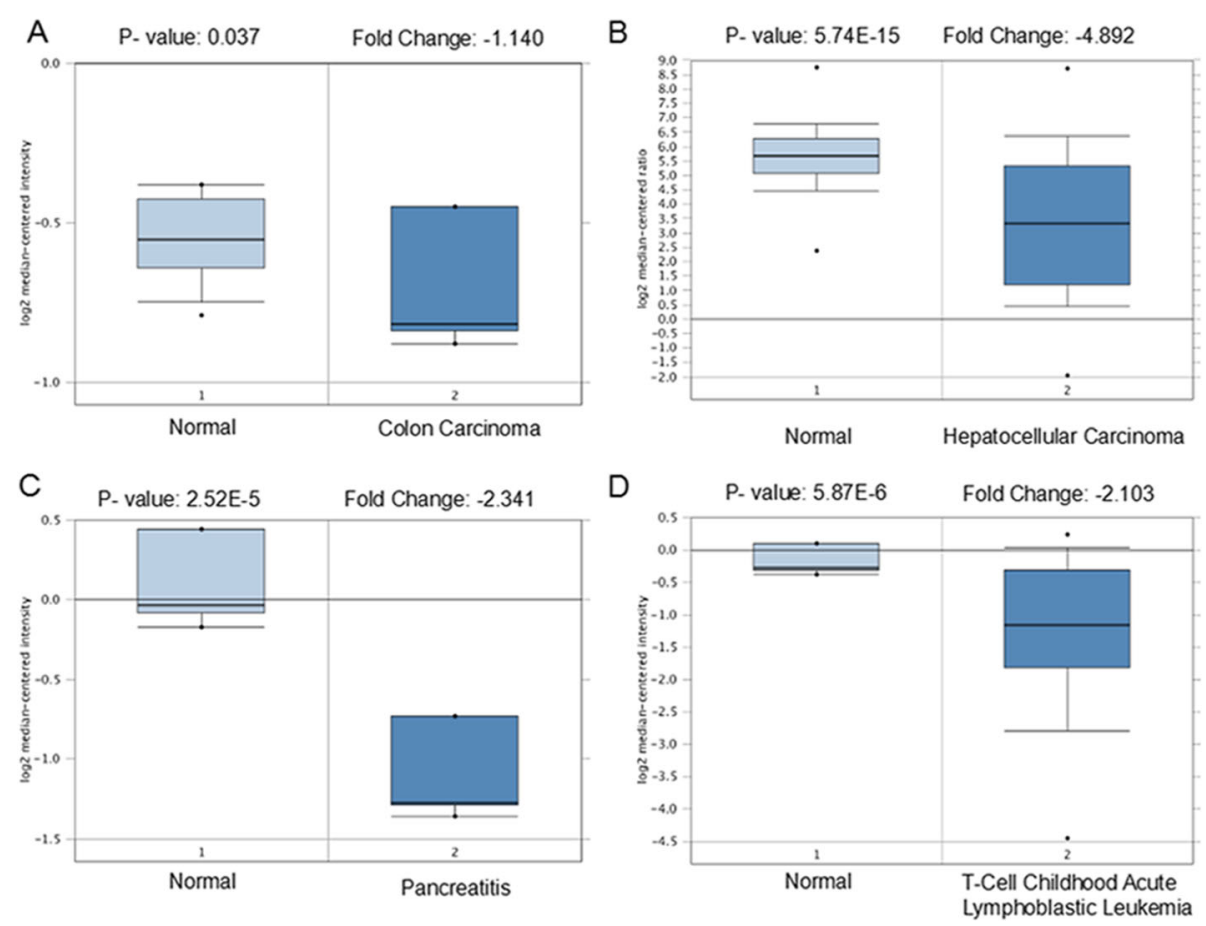

Fig. 3 The expression level of SPP2 in different cancer types (Oncomine database), The box plot comparing specific SPP2 expression in normal (left plot) and cancer tissue (right plot) was derived from Oncomine database. SPP2 was under-expressed in colon carcinoma (a), hepatocellular carcinoma (b), pancreatic cancer (c), and leukemia (d). The fold change of SPP2 in various types of cancers was identified from our analyses in Table 2

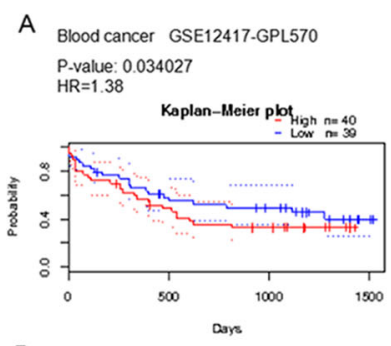

D Colorectal cancer GSE17536 P-value: 0.020234

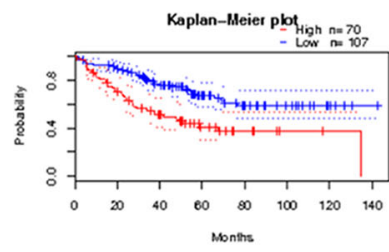

B Brain cancer GSE4412-GPL96
P-value: 0.001376
HR $=2.44$
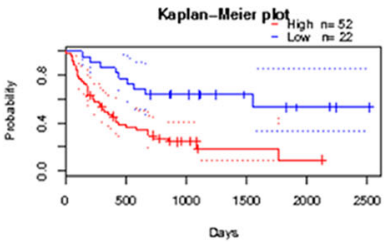

$E$

Lung cancer GSE31210 P. value: 0.000616 $\mathrm{HR}=1.96$

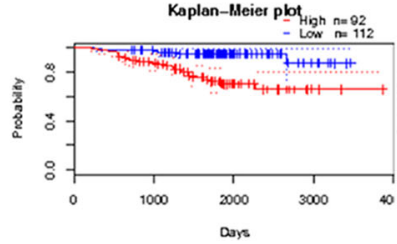
P-value 0.032468
Bret $H R=1.22$

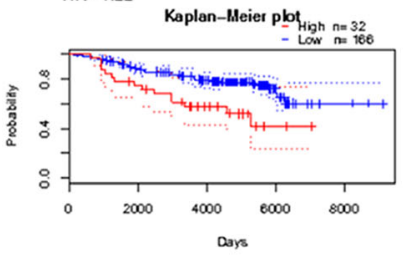

F

Melanoma GSE19234 P. value: 0.043445 $\mathrm{HR}=2.60$

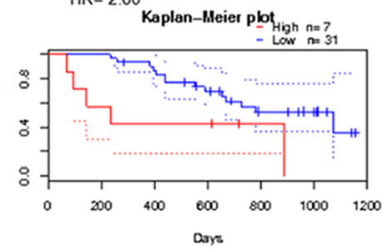

Fig. 4 The association between the expression of SPP1 gene and prognosis in blood cancer, brain cancer, breast cancer, colorectal cancer, lung cancer and melanoma (PrognoScan database), The survival curve comparing the patient with high (red) and low (blue) expression was plotted from PrognoScan database. The survival curve comparing the patient with high (red) and low (blue) expression in blood cancer (a), brain cancer (b), breast cancer (c), colorectal cancer (d), lung cancer $(\mathbf{e})$ and melanoma (f) was plotted from PrognoScan database as the threshold of cox $p$-value $<0.05$ 
Table 3 The association of SPP1 expression and the survival in cancer patients

\begin{tabular}{|c|c|c|c|c|c|c|}
\hline Cancer & $\mathrm{N}$ & Cox $p$-value & $\mathrm{HR}$ & Endpoint & Dataset & Probe ID \\
\hline Bladder & 165 & 0.027573 & 1.24 & Disease Specific Survival & GSE13507 & ILMN_1651354 \\
\hline Blood & 79 & 0.034027 & 1.38 & Overall Survival & GSE12417-GPL570 & 209875_s_at \\
\hline \multirow[t]{3}{*}{ Brain } & 50 & 0.011310 & 1.32 & Overall Survival & MGH-glioma & 34342_s_at \\
\hline & 50 & 0.014265 & 1.27 & Overall Survival & MGH-glioma & 2092_s_at \\
\hline & 74 & 0.001376 & 2.44 & Overall Survival & GSE4412-GPL96 & 209875_s_at \\
\hline \multirow[t]{6}{*}{ Breast } & 155 & 0.049236 & 0.83 & Overall Survival & GSE9893 & 400 \\
\hline & 286 & 0.026936 & 1.27 & Distant Metastasis Free Survival & GSE2034 & 209875_s_at \\
\hline & 159 & 0.001286 & 1.71 & Overall Survival & GSE1456-GPL96 & 209875_s_at \\
\hline & 159 & 0.011567 & 1.5 & Relapse Free Survival & GSE1456-GPL96 & 209875_s_at \\
\hline & 159 & 0.005257 & 1.73 & Disease Specific Survival & GSE1456-GPL96 & 209875_s_at \\
\hline & 198 & 0.032468 & 1.22 & Overall Survival & GSE7390 & 209875_s_at \\
\hline \multirow[t]{12}{*}{ Colorectal } & 62 & 0.0411806 & 1.46 & Overall Survival & GSE12945 & 209875_s_at \\
\hline & 177 & 0.0077045 & 1.33 & Disease Specific Survival & GSE17536 & 209875_s_at \\
\hline & 177 & 0.0332507 & 1.34 & Overall Survival & GSE17536 & 1568574_x_at \\
\hline & 177 & 0.0144318 & 1.45 & Disease Specific Survival & GSE17536 & 1568574_x_at \\
\hline & 177 & 0.0202343 & 1.23 & Overall Survival & GSE17536 & 209875_s_at \\
\hline & 145 & 0.00140364 & 1.56 & Disease Free Survival & GSE17536 & 209875_s_at \\
\hline & 226 & 0.00135618 & 1.40 & Disease Free Survival & GSE14333 & 209875_s_at \\
\hline & 49 & 0.030453 & 1.45 & Disease Specific Survival & GSE17537 & 1568574_x_at \\
\hline & 55 & 0.0163347 & 1.31 & Overall Survival & GSE17537 & 209875_s_at \\
\hline & 55 & 0.00149779 & 1.52 & Disease Free Survival & GSE17537 & 209875_s_at \\
\hline & 49 & 0.0310856 & 1.38 & Disease Specific Survival & GSE17537 & 209875_s_at \\
\hline & 55 & 0.000282164 & 1.72 & Disease Free Survival & GSE17537 & 1568574_x_at \\
\hline \multirow[t]{8}{*}{ Lung } & 104 & 0.0122453 & 1.67 & Overall Survival & jacob-00182-MSK & 209875_s_at \\
\hline & 204 & 0.000247927 & 2.45 & Overall Survival & GSE31210 & 209875_s_at \\
\hline & 204 & $3.04 \mathrm{E}-06$ & 2.18 & Relapse Free Survival & GSE31210 & 209875_s_at \\
\hline & 204 & 0.000616439 & 1.96 & Overall Survival & GSE31210 & 1568574_x_at \\
\hline & 204 & 4.89E-05 & 1.77 & Relapse Free Survival & GSE31210 & 1568574_x_at \\
\hline & 138 & 0.0119677 & 1.23 & Relapse Free Survival & GSE8894 & 209875_s_at \\
\hline & 138 & 0.0214765 & 1.25 & Relapse Free Survival & GSE8894 & 1568574_x_at \\
\hline & 129 & 0.0395 & 1.51 & Overall Survival & GSE4573 & 209875_s_at \\
\hline Skin & 38 & 0.0434455 & 2.6 & Overall Survival & GSE19234 & 209875_s_at \\
\hline
\end{tabular}

\section{SPP co-expression profiles in different cancers}

Oncomine was used to analys SPP co-expression. SPP1 co-expression profiles were determined in 41 head-neck squamous cell carcinoma and 13 normal tissues. The results showed that SPP1 was overexpressed in patients with head-neck cancer, and the top 3 genes co-expressing with SPP1 were Matrix Metallopeptidase 9 (MMP9), Actin Related Protein 2/ 3 Complex Subunit 1B (ARPC1B) and Amyloid Beta Precursor Protein (APP) (Fig.8 a). The co-expression profile of SPP2 was identified in 22 hepatocellular carcinoma and 21 normal tissues. However, SPP2 was down-regulated in liver cancer. The top 3 genes coexpressing with SPP2 were Leukocyte Cell Derived Chemotaxin 2 (LECT2), Carbamoyl-Phosphate Synthase 1 (CPS1) and Ribokinase (Fig.8 b).

\section{Discussion}

To date, the detailed function and role of SPP in cancer development and metastasis are poorly understood. Therefore, we assessed, for the first time, the predictive value and expression patterns of SPP in various cancers. 

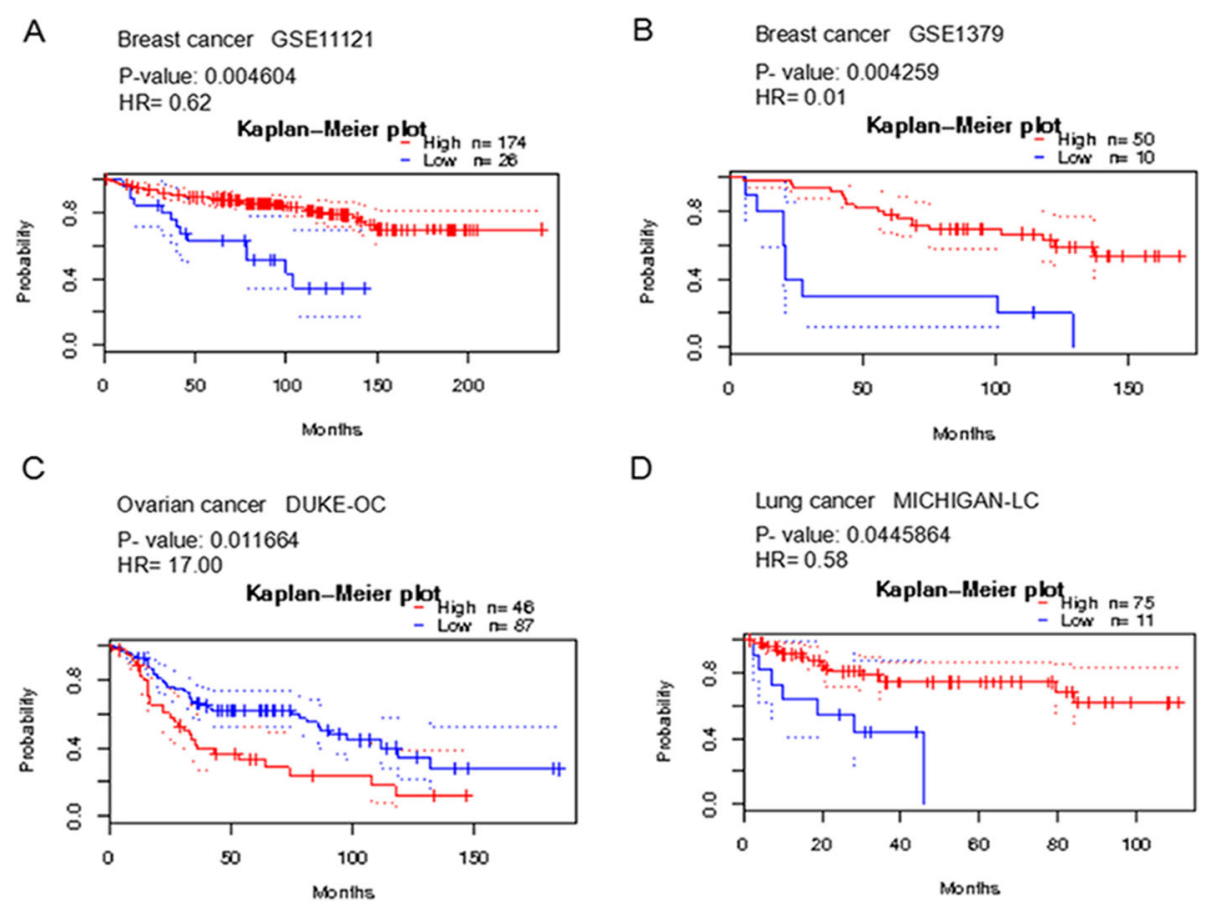

Fig. 5 The association between the expression of SPP2 and prognosis in Breast, Ovarian and Lung cancers (PrognoScan database), The survival curve comparing the patient with high (red) and low (blue) expression was plotted from PrognoScan database. The survival curve comparing the patient with high (red) and low (blue) expression in breast cancer $(\mathbf{a}, \mathbf{b})$, ovarian cancer $(\mathbf{c})$ and lung cancer (d) was plotted from PrognoScan database as the threshold of cox p-value $<0.05$

We are not able to define particular genes as tumor suppressor genes simply based on expression levels in the absence of explicit mechanistic studies, however, tapping into extensive oncogenic databases can provide researchers with a deeper understanding of the molecular mechanisms of these genes. Analysis of the relationship between SPP1 and the prognosis of various tumors revealed that SPP1 was upregulated in breast, bladder, colorectal, head and neck, liver, lung, and esophageal cancers. Further, high levels of SPP1 gene expression were associated with a poor prognosis for these cancers. SPP1 has been reported to enhance cancer cell survival, angiogenesis, and inflammation [15], while also promoting metastasis by favoring epithelial-to-mesenchymal transition [16]. This indicates that SPP1 is a tumorpromoting gene in these cancers. On the contrary, SPP2 was found to be deregulated in colorectal cancer, leukemia, liver cancer, and pancreatic cancer. Breast and lung cancer patients with high SPP2 expression had a better prognosis. BMPs contribute to the initiation and progression of multiple cancers. SPP2 has been shown to bind to BMP-2 and inhibit tumor growth through the blockage of BMP-2 [9]. Thus, SPP2 may have great potential as a clinical therapeutic agent.

Our SPP PPI network offers valuable insight into predicted interactions and functional relationships between genes. Most of the primary biological processes of SPP genes and the 10 predicted genes were related to the

Table 4 The association of SPP2 expression and the survival in cancer patients

\begin{tabular}{|c|c|c|c|c|c|c|}
\hline Cancer & $N$ & Cox $p$-value & $H R$ & Endpoint & Dataset & Probe ID \\
\hline \multirow[t]{2}{*}{ Breast } & 200 & 0.00460371 & 0.62 & Distant Metastasis Free Survival & GSE11121 & 214478_at \\
\hline & 60 & 0.00425913 & 0.01 & Relapse Free Survival & GSE1379 & 17,157 \\
\hline Colorectal & 177 & 0.0471794 & 0.18 & Disease Specific Survival & GSE17536 & 214478_at \\
\hline Lung & 86 & 0.0445864 & 0.58 & Overall Survival & MICHIGAN-LC & U20530_at \\
\hline Ovarian & 133 & 0.0116643 & 17.00 & Overall Survival & DUKE-OC & 214478_at \\
\hline
\end{tabular}




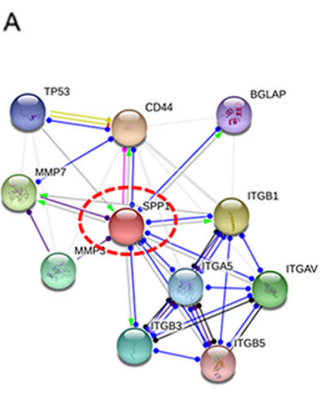

B

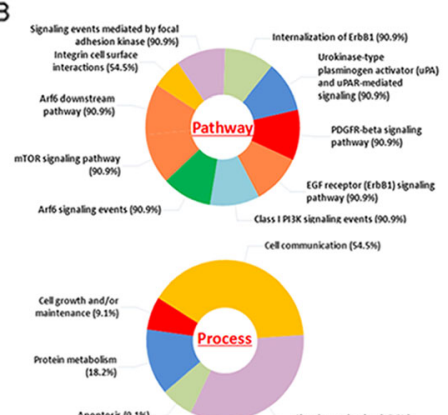

C

D
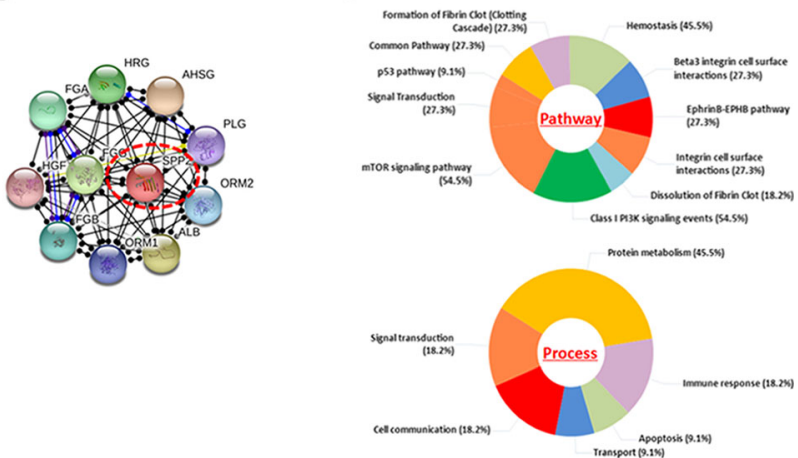

Fig. 6 Molecular functional pathways and process of SPP1 and SPP2, Interacting nodes are displayed in colored circles using String online tools $(\mathbf{a}, \mathbf{c})$. Pie chart for illustration of SPP1 (b) and SPP2 (d) molecular pathways and process was analyzed by Funrich software. Most of the primary biological processes of SPP genes and 10 predicted genes were the mTOR signaling pathway and class I PI3K signaling events, along with the main process of cell growth and maintenance, signaling transduction, cell communication, and protein metabolism

A

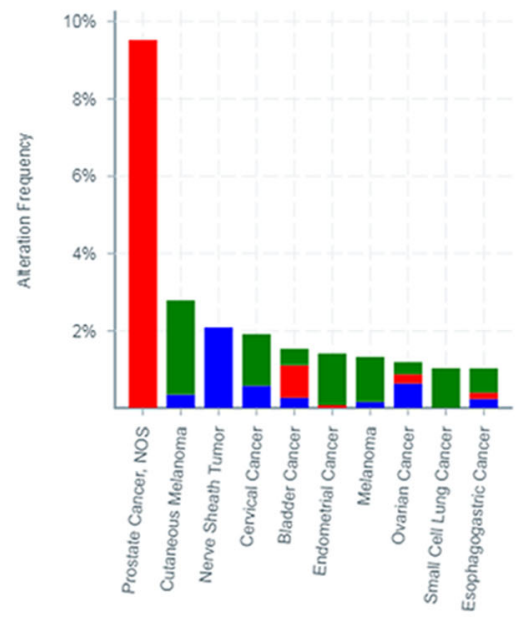

- Mutation - Amplification

- Deep Deletion
B

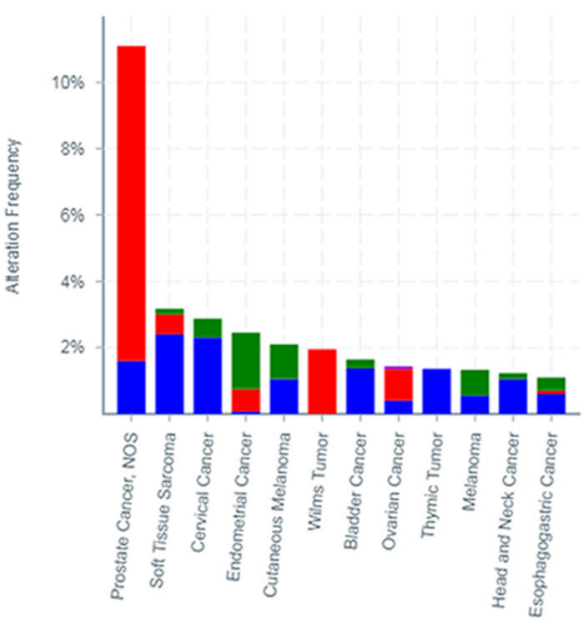

- Mutation - Fusion - Amplification - Deep Deletion

Fig. 7 Copy number alteration of SPP genes and cancer subtypes, The alteration frequency of SPP1 (a) and SPP2 (b) was determined by cBioPortal database. The alteration frequency included deletions (blue), amplification (red), Fusion (purple) or mutation (green) 
A

Ginos, Head-Neck Cancer

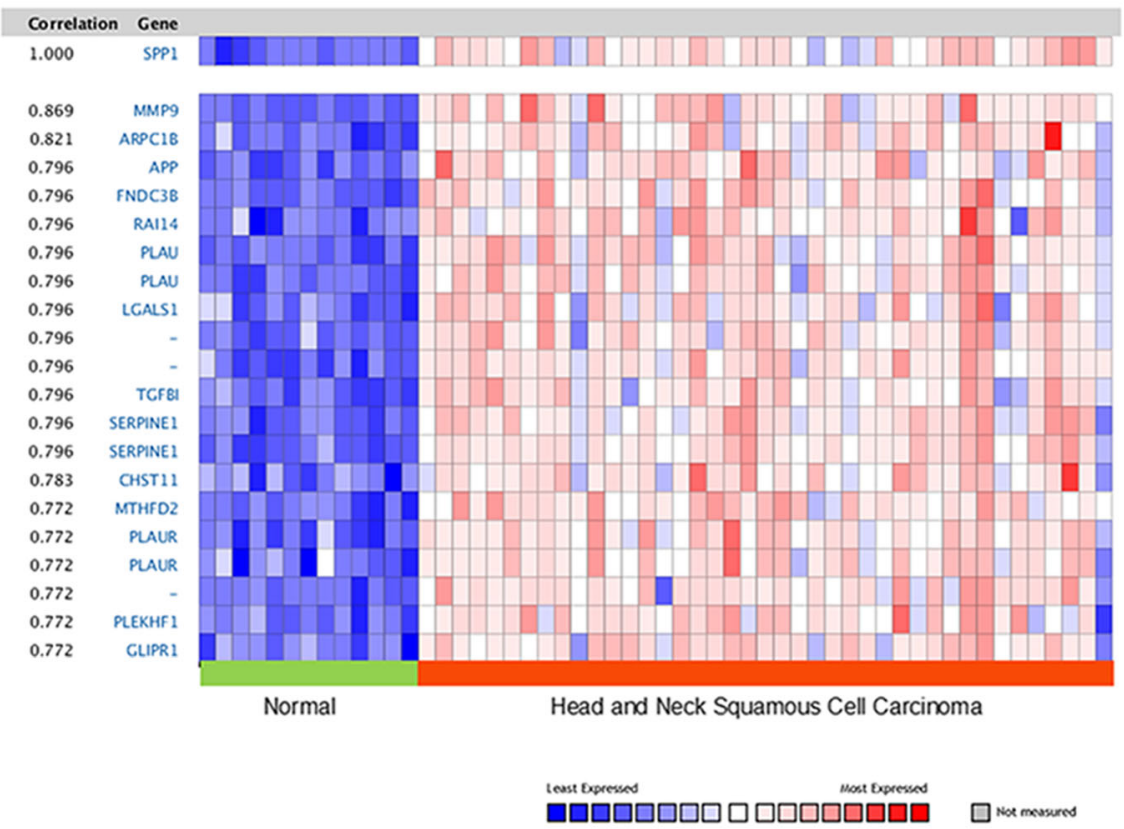

B Roessler, Hepatocellular Carcinoma

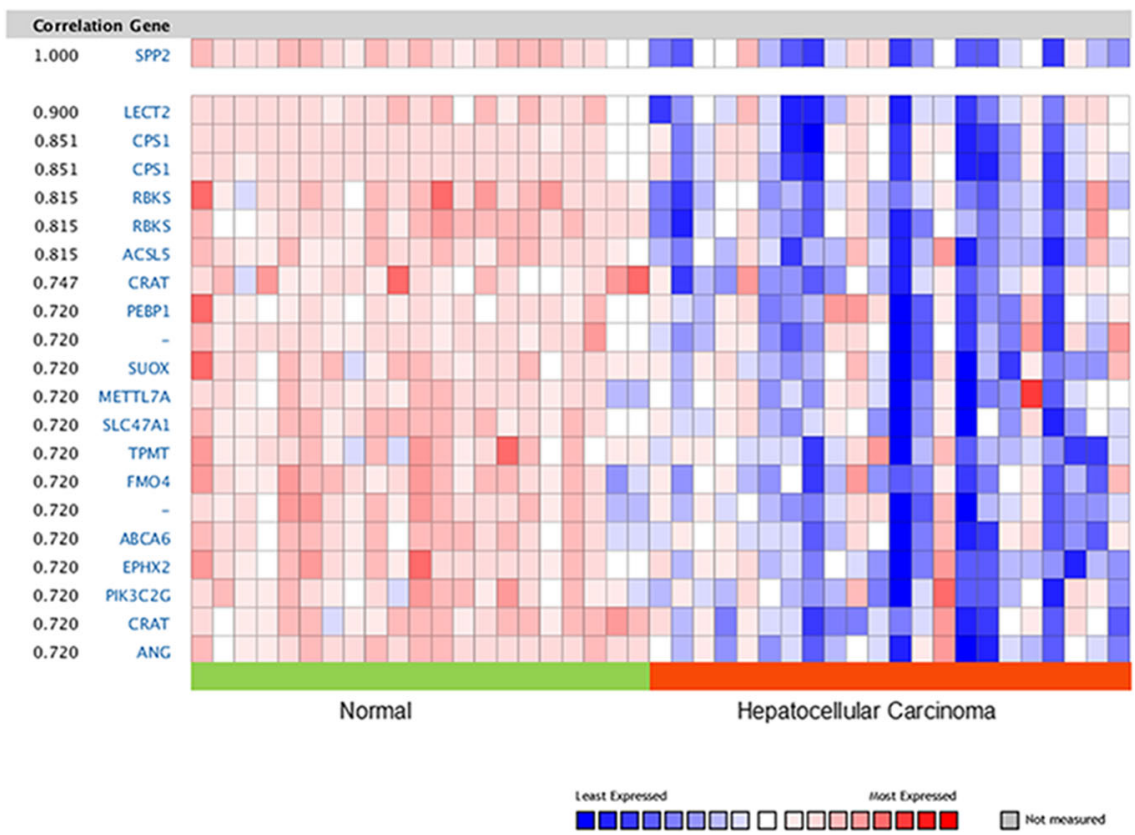

Fig. 8 Co-expression profiles of SPP genes in different types of cancers, (a) SPP1 genes in head-neck squamous cell carcinoma. SPP1 is coexpressed with the indicated genes across a panel of 41 head-neck squamous cell carcinoma and 13 normal tissues. Bar length represented the significance and negative logarithm of enrichment p-value. (b) SPP2genes in hepatocellular carcinoma.SPP2 is coexpressed with the indicated genes across a panel of 22 hepatocellular carcinoma and 21 normal tissues. Bar length represented the significance and negative logarithm of enrichment $p$-value

mTOR signaling pathway and class I PI3K signaling events, as well as cell growth and maintenance, signaling transduction, cell communication, and protein metabolism. Given the key role of the mTOR pathway in cell growth, regulation of actin cytoskeleton, gene transcription, ribosome biogenesis, and mRNA translation, there appears to be a link between mTOR activation and cancer. PI3K activation may be important for KRAS- 
induced tumorigenesis. Alteration of these pathways is strongly implicated in cancer pathogenesis; thus, targeting the effectors of these pathways is a promising therapeutic approach [17].

The cBioportal analysis revealed that SPP mutations and copy number alterations mainly occurred in prostate cancer, cutaneous melanoma, and endometrial cancer. It has been reported that somatically acquired genetics, epigenetics, transcriptomics, and proteomics are the main causes of cellular carcinogenesis. These changes may lead to suppression or carcinogenesis [18]. Coexpression analyses indicated that SPP1 was coexpressed with MMP9, ARPC1B, and APP in head-neck cancer. These results indicate that these genes have similar functions in head and neck cancer, and jointly promote tumorigenesis. SPP2 was co-expressed with LECT2, CPS1, and Ribokinase in hepatocellular carcinoma. This indicates that SPP2, LECT2, CPS1, and Ribokinase exert tumor suppressive effects in hepatocellular carcinoma.

\section{Conclusion}

To the best of our knowledge, the present study is the first to assess the importance and function of SPP family genes in the context of cancer development. Our systematic analysis of the expression and predictive value of SPP genes in various cancer types provided new insight into the heterogeneous expression of these genes in various cancers. Our findings indicate that SPP1 and SPP2 genes might play an important role in cancer progression. SPP1 appears to correlate with poor clinical outcomes, whereas SPP2 appears to be a potential prognostic marker for better survival. The inhibition or activation of SPP genes as a therapeutic approach for cancer treatment is dependent on the type of cancer to be treated. Further research is required to explore the signaling pathways and potential mechanisms of SPP genes in cancer and other diseases.

\section{Additional file}

Additional file 1: Figure S1. The association between the expression of SPP genes and prognosis in cancers (Kaplan- Meier Plotter database), The prognostic value of SPP1 and SPP2 expression level in breast cancer (A, C) and ovarian cancer (B, D) was plotted from Kaplan- Meier Plotter database.

\footnotetext{
Abbreviations

AHSG: Alpha 2-HS glycoprotein; ALB: Albumin; APP: Amyloid beta precursor protein; ARPC1B: Actin related protein 2/3 complex subunit 1B; BGLAP: Bone gamma-carboxyglutamate protein; BMPs: bone morphogenetic proteins; CAN: Copy number alterations; CPS1: Carbamoyl-phosphate synthase 1; FGA: Fibrinogen alpha chain; FGB: Fibrinogen beta chain; FGG: Fibrinogen gamma chain; HGF: Hepatocyte growth factor; HRG: Histidine rich glycoprotein; ITGA5: Integrin subunit alpha 5; ITGAV: Integrin subunit alpha V; ITGB1: Integrin subunit beta 1; ITGB3: Integrin subunit beta 3; ITGB5: Integrin subunit beta 5; LECT2: Leukocyte cell derived chemotaxin 2; MMP3: Matrix
}

metallopeptidase 3; MMP7: Matrix metallopeptidase 7; MMP9: Matrix metallopeptidase 9; ORM1: Orosomucoid 1; ORM2: Orosomucoid 2; PLG: Plasminogen; PPI: Protein-protein interaction; SPP1: Secreted phosphoprotein 1; SPP2: Secreted phosphoprotein 2; TP53: Tumor protein P53

\section{Acknowledgements}

The authors would like to thank all publicly available data used in the present study.

\section{Authors' contributions}

Conceived and designed the study strategy: GRF; Acquisition of data: statistical analysis and interpretation of data: YQT; Drafting or revision of the manuscript: YQT, GRF; Reference collection and data management: CC; Wrote the manuscript: YQT; Study supervision: GRF; All authors read and approved the final manuscript.

\section{Funding}

This work was supported by the National Natural Science Foundation of China (No. 81500796) and Research fund of Wuhan Union hospital (No.02.03.2017-321). The funding bodies played no role in the design of the study and collection, analysis, and interpretation of data and in writing the manuscript.

\section{Availability of data and materials}

The datasets generated and/or analyzed during the current study are available in the Oncomine database, [https://www.oncomine.org].

Ethics approval and consent to participate

Not applicable.

\section{Consent for publication}

Not applicable.

\section{Competing interests}

The authors declare that they have no competing interests.

\section{Author details}

'Department of Otorhinolaryngology, Union Hospital, Tongji Medical College, Huazhong University of Science and Technology, Wuhan 430022, China. ${ }^{2}$ Department of Endocrinology, The Central Hospital of Wuhan, Tongji Medical College, Huazhong University of Science and Technology, Wuhan, China.

Received: 6 May 2019 Accepted: 8 December 2019

Published online: 18 December 2019

\section{References}

1. Smith RA, Andrews KS, Brooks D, Fedewa SA, Manassaram-Baptiste D, Saslow D, Brawley OW, Wender RC. Cancer screening in the United States, 2017: a review of current American Cancer Society guidelines and current issues in cancer screening. CA Cancer J Clin. 2017;67(2):100-21.

2. Sadikovic B, Al-Romaih K, Squire JA, Zielenska M. Cause and consequences of genetic and epigenetic alterations in human cancer. Curr Genomics. 2008;9(6):394-408.

3. Cancer Genome Atlas Research N. Comprehensive genomic characterization of squamous cell lung cancers. Nature. 2012;489(7417):519-25.

4. Li S, Yang R, Sun X, Miao S, Lu T, Wang Y, Wo Y, Jiao W. Identification of SPP1 as a promising biomarker to predict clinical outcome of lung adenocarcinoma individuals. Gene. 2018;679:398-404.

5. Zhang Y, Du W, Chen Z, Xiang C. Upregulation of PD-L1 by SPP1 mediates macrophage polarization and facilitates immune escape in lung adenocarcinoma. Exp Cell Res. 2017;359(2):449-57.

6. Wei R, Wong JPC, Lyu P, Xi X, Tong O, Zhang SD, Yuen HF, Shirasawa S, Kwok HF. In vitro and clinical data analysis of Osteopontin as a prognostic indicator in colorectal cancer. J Cell Mol Med. 2018;22(9):4097-105.

7. Insua-Rodriguez J, Pein M, Hongu T, Meier J, Descot A, Lowy CM, De Braekeleer E, Sinn HP, Spaich S, Sutterlin M, et al. Stress signaling in breast cancer cells induces matrix components that promote chemoresistant metastasis. EMBO Mol Med. 2018;10(10).

8. Dayyani F, Zurita AJ, Nogueras-Gonzalez GM, Slack R, Millikan RE, Araujo JC, Gallick GE, Logothetis CJ, Corn PG. The combination of serum insulin, 
osteopontin, and hepatocyte growth factor predicts time to castrationresistant progression in androgen dependent metastatic prostate cancer- an exploratory study. BMC Cancer. 2016;16:721.

9. Li CS, Tian H, Zou M, Zhao KW, Li Y, Lao L, Brochmann EJ, Duarte ME, Daubs MD, Zhou YH, et al. Secreted phosphoprotein 24 kD (Spp24) inhibits growth of human pancreatic cancer cells caused by BMP-2. Biochem Biophys Res Commun. 2015;466(2):167-72.

10. Lao L, Shen J, Tian H, Yao Q, Li Y, Qian L, Murray SS, Wang JC. Secreted Phosphoprotein 24 kD inhibits growth of human prostate Cancer cells stimulated by BMP-2. Anticancer Res. 2016;36(11):5773-80.

11. Lao L, Shen J, Tian H, Zhong G, Murray SS, Wang JC. Secreted phosphoprotein 24kD (Spp24) inhibits growth of hepatocellular carcinoma in vivo. Environ Toxicol Pharmacol. 2017;51:51-5.

12. Mizuno H, Kitada K, Nakai K, Sarai A. PrognoScan: a new database for metaanalysis of the prognostic value of genes. BMC Med Genet. 2009;2:18.

13. Gao J, Aksoy BA, Dogrusoz U, Dresdner G, Gross B, Sumer SO, Sun Y, Jacobsen $A$, Sinha R, Larsson $E$ et al: Integrative analysis of complex cancer genomics and clinical profiles using the cBioPortal. Sci Signal 2013, 6(269):pl1.

14. Cerami E, Gao J, Dogrusoz U, Gross BE, Sumer SO, Aksoy BA, Jacobsen A, Byrne CJ, Heuer ML, Larsson E, et al. The cBio cancer genomics portal: an open platform for exploring multidimensional cancer genomics data. Cancer Discov. 2012;2(5):401-4

15. Hubbard NE, Chen QJ, Sickafoose LK, Wood MB, Gregg JP, Abrahamsson NM, Engelberg JA, Walls JE, Borowsky AD. Transgenic mammary epithelial osteopontin (spp1) expression induces proliferation and alveologenesis. Genes Cancer. 2013;4(5-6):201-12.

16. Xu C, Sun L, Jiang C, Zhou H, Gu L, Liu Y, Xu Q. SPP1, analyzed by bioinformatics methods, promotes the metastasis in colorectal cancer by activating EMT pathway. Biomed Pharmacother = Biomed Pharmacother. 2017:91:1167-77.

17. Wong KK, Engelman JA, Cantley LC. Targeting the PI3K signaling pathway in cancer. Curr Opin Genet Dev. 2010;20(1):87-90.

18. Klonowska K, Czubak K, Wojciechowska M, Handschuh L, Zmienko A, Figlerowicz M, Dams-Kozlowska H, Kozlowski P. Oncogenomic portals for the visualization and analysis of genome-wide cancer data. Oncotarget. 2016;7(1):176-92.

\section{Publisher's Note}

Springer Nature remains neutral with regard to jurisdictional claims in published maps and institutional affiliations.

Ready to submit your research? Choose BMC and benefit from:

- fast, convenient online submission

- thorough peer review by experienced researchers in your field

- rapid publication on acceptance

- support for research data, including large and complex data types

- gold Open Access which fosters wider collaboration and increased citations

- maximum visibility for your research: over $100 \mathrm{M}$ website views per year

At $\mathrm{BMC}$, research is always in progress.

Learn more biomedcentral.com/submissions 